\title{
Desempenho de Trichogramma pretiosum Riley e T. exiguum Pinto \& Platner (Hymenoptera: Trichogrammatidae) submetidos a diferentes densidades de ovos de Plutella xylostella (Lepidoptera: Plutellidae)
}

\author{
Performance of Trichogramma pretiosum Riley and T. exiguum Pinto \& Platner (Hymenoptera: \\ Trichogrammatidae) submitted to the eggs different densities \\ of Plutella xylostella (Lepidoptera: Plutellidae)
}

\author{
Fabricio Fagundes Pereira ${ }^{1}$ Reginaldo Barros ${ }^{2}$ Dirceu Pratissoli ${ }^{3}$
}

\section{RESUMO}

\begin{abstract}
Este trabalho teve como objetivo avaliar o desempenho de Trichogramma pretiosum Riley $e$ de Trichogramma exiguum Pinto \& Platner submetidos a diferentes densidades de ovos de Plutella xylostella (L.). Para cada espécie, grupos compostos por uma, duas e quatro fêmeas do parasitóide, foram confinadas em tubos de vidro juntamente com 15, 30, 45 e 60 ovos de P. xylostella por $24 \mathrm{~h}$ A duração do ciclo (ovo-adulto) foi uniforme e exatamente igual a nove dias, tanto para $\boldsymbol{T}$. pretiosum como para $\boldsymbol{T}$. exiguum. A porcentagem de parasitismo de $\boldsymbol{T}$. pretiosum e $\boldsymbol{T}$. exiguum mais elevada foi encontrada nas combinações de 15 ovos quatro fêmeas ${ }^{-1} e$ duas fêmeas 15 ovos $^{-1}$ respectivamente. A viabilidade do parasitismo para $\boldsymbol{T}$. pretiosum e T. exiguum não foi afetada pelas combinações de parasitóides e ovos para ambas as espécies. A razão sexual foi igual a um para T. pretiosum e variando de 0,6 a 0,8 para T. exiguum. $O$ número de parasitóides emergidos por ovo, de modo geral, foi um. As maiores porcentagens de deformação foram de 33,9 e 17,5\% dos descendentes para T. pretiosum $e$ T. exiguum, nos tubos contendo quatro fêmeas para 15 ovos, respectivamente. A combinação mais indicada para $\boldsymbol{T}$. pretiosum e T. exiguum foi uma fêmea 25 ovos $^{-1}$ e duas fêmeas 15 ovos $^{-1}$, respectivamente.
\end{abstract}

Palavras-chave: traça-das-crucíferas, controle biológico, parasitóide de ovos, taxa de parasitismo.

\section{ABSTRACT}

The objective of this research was to evaluate the performance of Trichogramma pretiosum Riley and Trichogramma exiguum Pinto \& Planter on different densities of Plutella xylostella eggs (L.). For both species, groups were made with one, two and four females of Trichogramma, and each group was confined in glass tubes with 15, 30, 45 and 60 eggs of $\boldsymbol{P}$. xylostella for 24 hours. Cycle duration (adult egg) of T. pretiosum and T. exiguum was around nine days. The highest parasitism rate of $\boldsymbol{T}$. pretiosum and $\boldsymbol{T}$. exiguum were reached when it used 15 eggs four females $^{-1}$ and 15 eggs two females ${ }^{-1}$, respectively. The viability of parasitism for $\boldsymbol{T}$. pretiosum and T. exiguum was not affected by the combinations of parasitoids and eggs for both species. The sex ratio was one for T. pretiosum and it varied from 0.6 to 0.8 to T. exiguum. The number of emerged individuals per egg was one. The greatest deformation rate of descendants for both species were observed in tubes containing four female $15 \mathrm{eggs}^{-1}$ with 33.9 for $\boldsymbol{T}$. pretiosum and 17.5 for $\boldsymbol{T}$. exiguum, respectively. The most indicated combination for $\boldsymbol{T}$. pretiosum and T. exiguum, was one female for $15 \mathrm{eggs}^{-1}$ and two famales $15 \mathrm{eggs}^{-1}$, respectively.

Key words: diamondback moth, biological control, eggs parasitoid, parasitism rate.

\section{INTRODUÇÃO}

Os insetos pertencentes ao gênero Trichogramma têm sido muito estudados, visando sua utilização no controle biológico de pragas de diversas plantas cultivadas. A crescente utilização desses insetos em liberações inundativas se deve principalmente à facilidade de criação massal ao baixo custo de produção e à comprovada eficiência no controle de lepidópteros-praga (HAJI et al., 2002).

Um aspecto considerado decisivo para o sucesso de liberações de parasitóides em campo é o conhecimento da proporção adequada de parasitóides liberados em relação à densidade de ovos do

\footnotetext{
${ }^{1}$ Engenheiro Agrônomo, Doutorando em Entomologia, Universidade Federal de Viçosa (UFV), Rua Maria das Neves de Jesus, nº 152, Bairro de Fátima, Edifício Fátima, Apt. 101, 36570 000, E-mail: ffpereira@insecta.ufv.br. Autor para correspondência. ${ }^{2}$ Engenheiro Agrônomo, Doutor, Professor de Entomologia, Departamento de Agronomia, Fitossanidade, Universidade Federal Rural de Pernambuco (UFRP), Avenida Dom Manuel de Medeiros s/n, Dois Irmãos, 52171-900, Recife, PE. E-mail: rbarros@ufrpe.br. ${ }^{3}$ Engenheiro Agrônomo, Doutor, Professor de Entomologia, Centro de Ciências Agrárias da Universidade Federal do Espírito Santo (UFES), Caixa Postal 16, 29500-000, Alegre, ES. E-mail:dirceu@npd.ufes.br.
} 
hospedeiro presente em um determinado agroecossistema, pois a eficiência dos mesmos pode ser reduzida, devido à competição intra-específica, uma vez que à medida que se aumenta a densidade de parasitóides, decresce a probabilidade de um indivíduo encontrar um ovo não-parasitado, podendo neste caso ocorrer superparasitismo (NEIL \& SPECHT, 1990).

A relação adequada entre o número de parasitóides e ovos do hospedeiro pode variar de acordo com a espécie, a idade e o tempo de exposição do hospedeiro (FARIA et al., 2000). Segundo PARRA(1997), esta relação não pode ser muito elevada (superparasitismo) nem muito baixa, pois ambas as situações poderão ocasionar deficiências na eficiência dos parasitóides, comprometendo o desempenho dos mesmos.

Em criações massais de Trichogramma, nas quais esses insetos são mantidos em altas densidades, é comum se observarem efeitos deletérios nas progênies decorrentes do superparistismo, como a presença de deformações, redução na capacidade de busca, decréscimo na fecundidade e na razão sexual (KFIR, 1981; 1988).

Espécies como Trichogramma pretiosum Riley e Trichogramma exiguum Pinto \& Platner têm sido mencionadas em diversos países como importante alternativa para o controle de Plutella xylostella (L.) (HASSAN, 1997). No Brasil, são poucas as pesquisas relacionando esses parasitóides a $\boldsymbol{P}$. xylostella (BARROS \& VENDRAMIM, 1999), sendo este o primeiro registro de T. exiguum associado a esta praga.

Desta forma, estudos estabelecendo a adequada proporção entre o número de fêmeas de T. pretiosum e T. exiguum e ovos de P. xylostella precisam ser realizados, pois os mesmos fornecem informações sobre parâmetros comportamentais envolvidos no processo de parasitismo, que são considerados relevantes para pesquisas posteriores de laboratório e campo, que poderão proporcionar condições para que o potencial desses agentes de controle biológico sejam efetivamente alcançados.

O objetivo deste trabalho foi avaliar o desempenho de $\boldsymbol{T}$. pretiosum e de $\boldsymbol{T}$. exiguum submetidos a diferentes densidades de ovos de $\boldsymbol{P}$. xylostella.

\section{MATERIAL E MÉTODOS}

O experimento foi conduzido no Laboratório de Entomologia do Centro de Ciências Agrárias da Universidade Federal do Espírito Santo (CCA-UFES) à temperatura de $25 \pm 1^{\circ} \mathrm{C}$, umidade relativa de $70 \pm 10 \%$ e fotofase de 14 horas.
Criação de $\boldsymbol{P}$. xylostella

Inicialmente cerca de 500 pupas de $\boldsymbol{P}$. xylostella, provenientes da criação estoque do laboratório de Biologia de Insetos da Área de Fitossanidade da (UFRPE), foram transferidas para o laboratório de Entomologia do (CCA-UFES), onde foi iniciada a criação desta praga segundo metodologia adotada por BARROS \& VENDRAMIN (1999).

Manutenção e multiplicação dos parasitóides

A espécie T. pretiosum foi proveniente da criação estoque do laboratório de Entomologia do (CCA-UFES), e inicialmente foi coletada em plantios comerciais de tomate localizados no município de Alegre, ES. Já a espécie T. exiguum foi coletada também em plantios de tomate, porém no município de Muniz Freire, ES. As duas espécies foram enviadas para R.A. Zucchi, do Departamento de Entomologia da Escola Superior de Agricultura “Luiz de Queiroz" Piracicaba, SP, para identificação.

Para a manutenção dos parasitóides, ovos de A. kuehniella, obtidos segundo técnica adaptada de PARRA (1997), foram inviabilizados e colados com goma arábica diluída a $30 \%$ em retângulos de cartolina azul celeste $(8,0 \times 2,0 \mathrm{~cm})$. Estas cartelas foram inseridas em tubos de vidro $(8,5 \times 2,4 \mathrm{~cm})$, contendo adultos recém-emergidos, e em seguida os tubos foram vedados com filme de PVC, a fim de se evitar a fuga dos parasitóides. Na multiplicação, foi adotado o mesmo procedimento para manutenção, alterando-se apenas o tamanho do retângulo para $10 \mathrm{x}$ $10 \mathrm{~cm}$, a fim de se evitar o superparasitismo. Desse modo, foi permitido o parasitismo por 24 horas em sala climatizada, á temperatura de $25 \pm 1^{\circ} \mathrm{C}$, umidade relativa de $70 \pm 10 \%$ e fotofase de 14 horas.

Efeito da densidade de ovos de $\boldsymbol{P}$. xylostella no desempenho de $T$. pretiosum e T. exiguum.

Fêmeas de T. pretiosum e T. exiguum com 12 a 24 horas de idade foram individualizadas, para a composição de grupos com uma, duas e quatro fêmeas, que foram introduzidas em tubos de vidro $(3,0 \times 0,4 \mathrm{~cm})$ contendo cartelas de cartolina azul celeste $(2,5 \times 0,3 \mathrm{~cm})$ com 15, 30, 45 e 60 ovos do dia de P. xylostella, os quais foram coletados, com auxílio de um pincel de pêlos finos, de discos de couve provenientes da criação-estoque de $\boldsymbol{P}$. $x$ ylostella. Essas cartelas foram expostas ao parasitóide por 24 horas em câmara climatizada, regulada a $25 \pm 1^{\circ} \mathrm{C}, 70 \pm 10 \%$ de umidade relativa e fotofase de 14 horas. Ao final desse período, as fêmeas foram retiradas dos tubos com auxílio de microscópio estereoscópico. Após a duração do ciclo (ovo-adulto) dos parasitóides no hospedeiro, foram 
avaliados os seguintes parâmetros biológicos: duração do ciclo (ovo-adulto); porcentagem de parasitismo; número de ovos parasitados (escurecidos); viabilidade do parasitismo; número de parasitóides emergidos por ovo de $\boldsymbol{P}$. xylostella; razão sexual e porcentagem de deformação.

O experimento foi conduzido no esquema fatorial 3 x 4 (três níveis do parasitóide e quatro níveis do hospedeiro) com 20 repetições no delineamento inteiramente casualizado. Os resultados foram analisados, estatisticamente, em nível de significância de $5 \%$ de erro, sendo os valores significativos submetidos à análise de regressão.

Para a análise de regressão, adotou-se a metodologia de superfície de resposta, a partir dos modelos linear e quadrático com duas variáveis independentes, dado por:

$$
\begin{aligned}
& \text { Linear } \\
& Y=\beta_{0}+\beta_{1} X_{1}+\beta_{2} Z_{1}+e_{i} \text { onde } \quad Y=\beta_{0}+\beta_{1} X_{1}+\beta_{2} X^{2}{ }_{1}+\beta_{3} Z_{1}+ \\
& \mathrm{e}_{\mathrm{i}} \text { onde } \\
& \mathrm{Y}=\text { porcentagem de parasitismo; } \\
& \mathrm{Y}=\text { porcentagem de parasitismo; } \\
& \mathrm{X}_{1}=\text { número de fêmeas: } \\
& \mathrm{Z}_{1}=\text { densidade de ovos; } \\
& \beta_{\mathrm{i},} \text { com } \mathrm{i}=0,1 \text { e } 2=\text { parâmetros } \\
& \text { estimados; } \\
& \mathrm{e}_{\mathrm{i}}=\text { erro aleatório. } \\
& \mathrm{X}_{1}=\text { número de fêmeas; } \\
& \mathrm{Z}_{1}=\text { densidade de ovos; }
\end{aligned}
$$

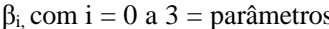

$$
\begin{aligned}
& \text { estimados; } \\
& \mathrm{e}_{\mathrm{i}}=\text { erro aleatório. }
\end{aligned}
$$

A partir dos modelos apresentados acima, a escolha da equação que melhor se ajustou aos dados foi com base no coeficiente de determinação $\left(\mathrm{R}^{2}\right)$, na significância da regressão pelo teste $\mathrm{F}$ (até $5 \%$ de probabilidade).

\section{RESULTADOS E DISCUSSÃO}

A duração do ciclo (ovo-adulto) foi uniforme e exatamente igual a nove dias, tanto para $\boldsymbol{T}$. pretiosum como para T. exiguum, portanto não foi afetada pelas combinações envolvendo densidades de parasitóides e número de ovos da praga. Já a porcentagem de parasitismo para ambas as espécies de parasitóides foi influenciada pelas combinações de número de fêmeas com densidades de ovos, como pode ser observada nas (Figuras 1 e 2), em que foram estabelecidas equações de regressão e suas respectivas superfícies de resposta.

Quando se confinou uma, duas e quatro fêmeas de T. pretiosum por tubo, observou-se declínio na porcentagem de parasitismo com respostas lineares à medida que se aumentou o número de ovos de 15 para 60 (Figura 1), evidenciando a influência negativa do excesso de ovos à disposição da fêmea, fato também observado por PARON et al. (1998) estudando $\boldsymbol{T}$. atopovirilia Oatman \& Platner em ovos de
Helicoverpa zea (Boddie), resultados que podem ser decorrentes da competição entre as fêmeas e ao fato do período de 24 horas ser insuficiente para que houvesse maior parasitismo.

Dentre as combinações estudadas, os maiores valores de porcentagem de parasitismo foram encontrados quando se confinaram quatro fêmeas de T. pretiosum na densidade de 15 ovos fêmea ${ }^{-1}$, entretanto não se recomenda esta combinação, por ter ocorrido superparasitismo, ou seja, ovos foram parasitados mais de uma vez, e por conseqüência houve competição entre larvas desse parasitóide por alimento, originando indivíduos menores e deformados. Como níveis elevados de parasitismo também foram obtidos na faixa entre 15 e 30 ovos fêmea $^{-1}$, pode-se inferir que a relação de uma fêmea para 25 ovos de $\boldsymbol{P}$. xylostella pode ser considerada como ideal (Figura 1).

Em relação à $\boldsymbol{T}$. exiguum, observou-se que a porcentagem de parasitismo em função das combinações de uma duas e quatro fêmeas com 15, 30, 45 e 60 ovos, mostrou um comportamento quadrático. Este parâmetro biológico foi mais elevado quando se colocaram duas fêmeas por tubo na densidade de 15 ovos, havendo um declínio do parasitismo quando se utilizou uma e quatro fêmeas nas demais densidades de ovos (Figura 2). Supõe-se que uma fêmea não foi suficiente para parasitar 15 ovos e quatro fêmeas superparitaram os ovos de $\boldsymbol{P}$. xylostella e que este último comportamento pode ser atribuído à alta agressividade das fêmeas de $\boldsymbol{T}$. exiguum, pois essa espécie possui apenas sete gerações em laboratório e, portanto suas características de campo estão predominando com maior intensidade. De acordo com VINSON (1997), há vários fatores que podem influenciar na intensidade de procura, aceitação, oviposição e marcação do hospedeiro, como a quantidade de ovos disponíveis, a presença de outras fêmeas e número de gerações da espécie de Trichogramma em laboratório.

Resultados semelhantes foram encontrados por KFIR (1981), quando avaliou o efeito do número de ovos de Heliothis spp. sobre T. pretiosum. Este autor observou que, com o aumento da densidade do parasitóide de uma para quatro fêmeas 300 ovos $^{-1}$, houve um decréscimo de $50 \%$ de ovos parasitados. Também constatou um aumento de $2,4 \%$ na taxa de parasitóides de menor tamanho para $12,4 \%$ e queda nas proporções de fêmeas de $72 \%$ para $39 \%$, indicando claramente os efeitos adversos da superpopulação do parasitóide para liberações inundativas.

De maneira geral, a viabilidade do parasitismo para $\boldsymbol{T}$. pretiosum e T. exiguum, não foi afetada pelas combinações de parasitóides e ovos, 


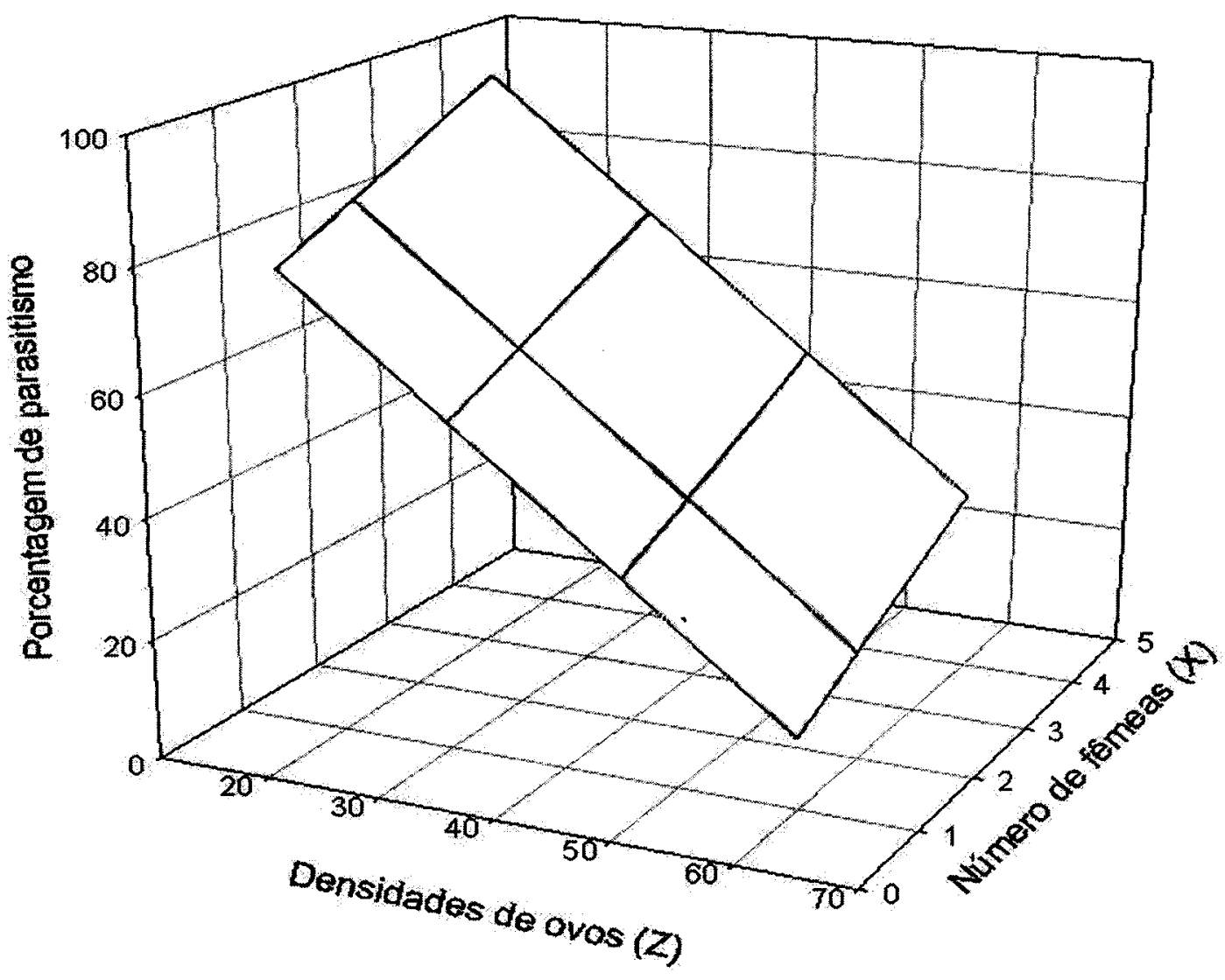

$Y=90,68+6,18 X-1,42 Z\left(R^{2}=0,96^{* *}\right)$

Figura 1- Relação entre a densidade de ovos de $\boldsymbol{P}$. xylostella, número de fêmeas e a porcentagem de parasitismo de $\boldsymbol{T}$. pretiosum a $25 \pm 1^{\circ} \mathrm{C}, 70 \pm 10 \%$ de UR e fotofase de 14 horas.

mantendo-se valores no intervalo entre 88,7 e $97,9 \%$ para ambas as espécies. A razão sexual também foi igual a 1 para $\boldsymbol{T}$. pretiosum e variou de 0,6 a 0,8 para $\boldsymbol{T}$. exiguum, evidenciando que, por se tratar de uma espécie criada há bastante tempo em laboratório, $\boldsymbol{T}$. pretiosum encontra-se melhor adaptada do que $\boldsymbol{T}$. exiguum, criada apenas por sete gerações.

Quanto ao número de parasitóides emergidos por ovo de $\boldsymbol{P}$. xylostella, observou-se a predominância de um parasitóide, existindo, porém a ocorrência de mais de um nas relações de quatro parasitóides para 15 ovos, demonstrando a ocorrência de superparasitismo.

A porcentagem de deformações de adultos foi nula nas combinações até dois parasitóides, para ambas as espécies. Ocorreu, entretanto, nas combinações de quatro parasitóides para 15, 30 e 45 ovos de $\boldsymbol{P}$. xylostella com 17,5; 10,0 e 2,5\%, respectivamente. Fato esse creditado possivelmente ao superparasitismo, evidenciando que as combinações com densidades mais elevadas resultaram numa redução da porcentagem de parasitismo, e por conseguinte, maiores percentuais de adultos deformados.

Vale ressaltar que estudos relacionados à idade e distribuição do hospedeiro na planta, número e intervalo de liberações dos parasitóides, além da capacidade de dispersão dos mesmos, ainda precisam ser realizados para que tais agentes de controle biológico possam expressar todo o seu potencial no controle de $\boldsymbol{P}$. xylostella em nível de campo.

\section{CONCLUSÕES}

A observação conjunta desses resultados evidencia que a relação de uma fêmea de $\boldsymbol{T}$. pretiosum 


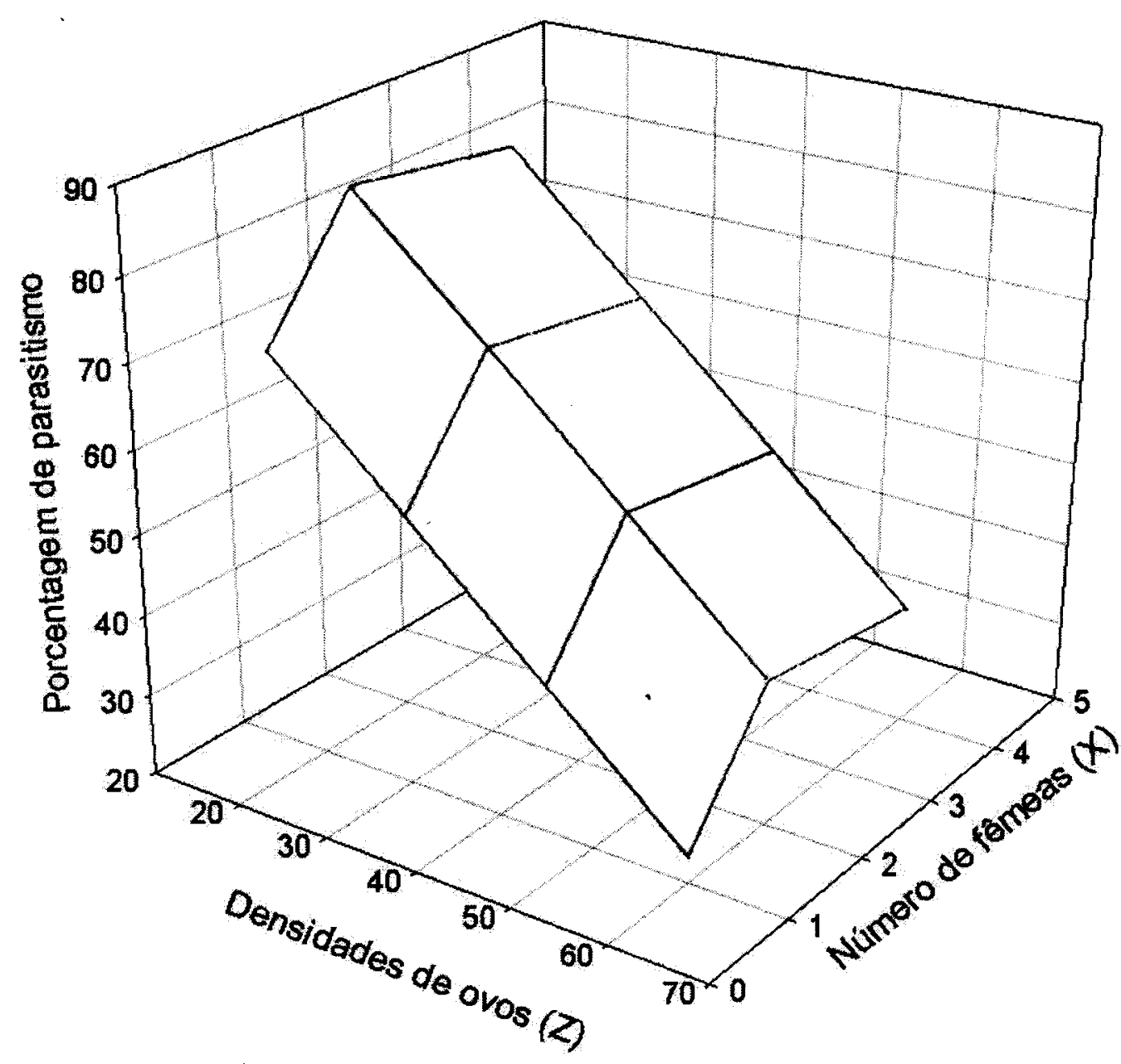

\section{$Y=57,14+31,28 X-5,50 X^{2}-0,99 Z\left(R^{2}=0,72^{\star}\right)$}

Figura 2 - Relação entre a densidade de ovos de $\boldsymbol{P}$. xylostella, número de fêmeas e a porcentagem de parasitismo de $\boldsymbol{T}$. exiguum a $25 \pm 1^{\circ} \mathrm{C}, 70 \pm 10 \%$ de UR e fotofase de 14 horas.

25 ovos $^{-1}$ de $\boldsymbol{P}$. xylostella é considerada ideal. Já para T. exiguum, a melhor combinação foi duas fêmeas 15 ovos $^{-1}$. As demais combinações não devem ser recomendadas por proporcionarem decréscimo na eficiência dos parasitóides, comprometendo o desempenho dos mesmos em criações massais.

\section{AGRADECIMENTOS}

À Coordenação de Aperfeiçoamento de Pessoa de Nível Superior - CAPES, pela concessão da bolsa de estudo ao primeiro autor; à Universidade Federal Rural de Pernambuco; e ao Laboratório de Entomologia do Centro de Ciências
Agrárias da Universidade Federal do Espírito Santo (CCAUFES), por possibilitarem a execução desta pesquisa.

\section{REFERÊNCIAS BIBLIOGRÁFICAS}

BARROS, R.; VENDRAMIN, J.D. Efeito de cultivares de repolho, utilizados para criação de Plutella xylostella (L.) (Lepidoptera: Plutellidae), no desenvolvimento de Trichogramma pretiosum Riley (Hymenoptera: Trichogrammatidae). Anais da Sociedade Entomológica do Brasil, Londrina, v.28, n.3, p.469-476, 1999.

FARIA, C.A. et al. Resposta funcional de Trichogramma pretiosum Riley (Hymenoptera: Trichogrammatidae) parasitando ovos de Tuta absoluta (Meyrick) (Lepidoptera: 
Gelechiidae): Efeito da idade do hospedeiro. Anais da Sociedade Entomológica do Brasil, Londrina, v.29, n.1, p.85-93. 2000.

HAJI, F.N.P. et al. Trichogramma pretiosum para o controle de pragas no tomateiro industrial, In: PARRA, J.R.P. et al. (eds.). Controle biológico no Brasil: parasitóides e predadores. São Paulo : Manole, 2002. Cap.28, p.477-494.

HASSAN, S.A. Seleção de espécies de Trichogramma para o uso em programas de controle biológico. In: PARRA, J.R.P.; ZUCCHI, R.A. Trichogramma e controle aplicado. Piracicaba : FEALQ, 1997. Cap.7, p.183-205.

KFIR, R. Effect of hosts parasite density on the egg parasite Trichogramma pretiosum. Entomophaga, Paris, v.26, n.1, p.445451, 1981.

KFIR, R. Funtional response to host density by the egg parasite Trichogramma pretiosum. Entomophaga, Paris, v.28, p.345-353, 1988 .

NEIL, K.A.; SPECHT, H.B. Field releases of Trichogramma pretiosum Riley (Hymenoptera:
Trichogrammatidae) for suppression of corn carworm, Heliothis zea (Boddie) (Lepidoptera: Noctuidae), egg populations on sweet corn in Nova Scotia. The Canadian Entomologist, Ottawa, v.122, n.3, p.1259-1266, 1990

PARON, M.J.F.O. et al. Resposta de Trichogramma atopovirilia Oatman \& Platner (Hymenoptera: Trichogrammatidae) a diferentes densidades de ovos do hospedeiro natural, Helicoverpa zea (Boddie) (Lepidoptera: Noctuidae). Anais da Sociedade Entomológica do Brasil, Londrina, v.27, n.3, p.427433, 1998 .

PARRA, J.R.P. Técnicas de criação de Anagasta kuehniella, hospedeiro alternativo para produção de Trichogramma. In: PARRA, J.R.P.; ZUCCHI, R.A. (eds.). Trichogramma e controle aplicado. Piracicaba: FEALQ, 1997. Cap.4, p.121-150.

VINSON, S.B. Comportamento de seleção hospedeira de parasitóides de ovos, com ênfase na família Trichogrammatidae. In: PARRA, J.R.P.; ZUCCHI, R.A. (eds.). Trichogramma e o controle aplicado. Piracicaba : FEALQ, 1997. Cap.3, p.67-119, 1997. 